\title{
Accuracy of Digital Impressions Achieved from Five Different Digital Impression Systems
}

\section{Ala Omar Ali}

Tufts University School of Dental Medicine, Boston, MA, USA

\begin{abstract}
Objective: This study aimed to compare the accuracy of digital impressions obtained from various digital impression systems.

Material and methods: A typodont was prepared for a three-unit bridge, and an epoxy resin model of this preparation was designed as a reference model. A lab scanner was then used to record a digital copy of the reference model. The different systems (3M Lava C.O.S., 3Shape D900, Cadent iTero, CEREC Bluecam, and E4D Dentist) were used to scan the epoxy resin reference model and create five digital impressions each $(n=5)$. Using computer software, the differences in spatial measurements between the digital reference model and digital impressions and were calculated. The accuracy was evaluated based on the mean difference and standard deviation in micrometers $(\mu \mathrm{m})$ for each system's set of five digital impressions.
\end{abstract}

Results: The measurements for mean difference (standard deviation) were as follows: CadentiTero-23 (3) $\mu \mathrm{m}, 3 \mathrm{M}$ Lava C.O.S. - 36 (19) $\mu \mathrm{m}$, 3Shape D900- 44 (18) $\mu \mathrm{m}$, CEREC Bluecam - 68 (12) $\mu \mathrm{m}$, E4D Dentist - 84 (4) $\mu \mathrm{m}$. The One Way ANOVA test was significant $(p \leq 0.001)$.Multiple comparison post-hoc tests showed that the E4D Dentist system was significantly different from the Cadent iTero, 3M Lava C.O.S., and 3ShapeD900systems. Also, CEREC Bluecam exhibited significant differences from Cadent iTeroand3M Lava C.O.S. At the same time, there were no significant differences between Cadent iTero, 3M Lava C.O.S., and 3ShapeD900.

Conclusion: Within the limitations of this study, the following conclusions were drawn:

1) There were statistically significant differences between the accuracy of the digital impression systems. More specifically, this difference was most notable when comparing the systems that achieved lower accuracy measurements versus those that demonstrated accuracy on the high end.

2) Digital impressions from the Cadent iTero system were the most accurate.

Clinical significance of the study: The results of this study could affect the decision of the clinician on selecting an appropriate Computer-Aided Design/Computer-Aided Manufacturing (CAD/CAM) scanner for digital impressions. Furthermore, the results carry implications of whether digital impressions are accurate enough to be used as an alternative to conventional impression techniques.

Keywords: Digital impressions; Master models; Lava COS; iTero; CEREC 3D; E4D; 3Shape

\section{Introduction}

Fabrication of final dental restorations through conventional practices involves a complicated process. The accuracy of the final restoration may be affected by factors such as tooth preparation design, final impression technique, master cast production, fabrication of the restoration, chair side adjustment of the restoration, and the material and method used for cementation [1-4].

The impression technique, in addition to the properties of the particular impression material that is used, may ultimately affect the fit of fixed restorations. The fabrication of a stone master cast compensates for volumetric changes of the impression material to a certain extent; however, the fabrication of a master cast is a time-consuming and error-prone method that requires the services of a dental laboratory $[5,6]$.

A relatively new approach employs Computer-Aided Design/ Computer-Aided Manufacturing (CAD/CAM) technology to take a digital impression intra orally, fabricate the master model, and design as well as produce the final restoration. This method aims to overcome certain physical limitations of conventional means, such as the dimensional changes of impression materials, the expansion of dental stone, and human errors associated with final restoration fabrication, thus reducing processing time as well as cost [7]
Dr. Duret first introduced the CAD/CAM concept to dentistry in 1973 in Lyon, France in his thesis entitled Empreinte Optique, which translates to Optical Impression. The concept of CAD/CAM systems was further developed by Dr. Mormann, a Swiss Dentist, and Mr. Brandestini, who was an electrical engineer. 8 CEREC was the first commercially available digital impression system for use in the field of dentistry. Over the last 10 years, systems like 3M Lava C.O.S., Cadent iTero, E4D Dentist, and 3Shape Trios have been introduced. To date, various $\mathrm{CAD} / \mathrm{CAM}$ systems are now available for dental applications. Each employs a specific, distinct technique for making impressions [8].

Some CAD/CAM systems, like 3M Lava C.O.S. and CEREC Bluecam, require the application of a titanium dioxide or magnesium oxide powder to the abutment teeth before scanning them in order to eliminate reflection and create a measurable surface. The powder

*Corresponding author: Ala Omar Ali, Tufts University School of Dental Medicine, Boston, MA, One Kneeland St, Office 224, Boston, MA 02111, USA, Tel: 4074214607; E-mail: ala.ali@tufts.edu; alaaoali@gmail.com

Received February 04, 2015; Accepted April 21, 2015; Published April 24, 2015

Citation: Ali AO (2015) Accuracy of Digital Impressions Achieved from Five Different Digital Impression Systems. Dentistry 5: 300. doi:10.4172/2161-1122.1000300

Copyright: @ 2015 Ali AO. This is an open-access article distributed under the terms of the Creative Commons Attribution License, which permits unrestricted use, distribution, and reproduction in any medium, provided the original author and source are credited. 
layer on the tooth surface results in an additional thickness of 13-85 $\mu \mathrm{m}$ [9]. Other systems, like CEREC Omnicam, E4D Dentist, Cadent iTero, and 3Shape Trios, do not require this powder layer because the scanner software can handle the glossy surface of the abutment teeth. Regardless of the digitizing mode applied, clinical parameters such as saliva, blood, or movements of the patient can affect the accurate reproduction of teeth [9-12].

Another CAD/CAM method does not involve intraoral scanning, but it allows dental laboratory technicians to produce restorations using conventional impressions without the need for stone master models. This can be achieved through systems that scan the conventional impression materials and replicate them in digital form; an example of a device capable of doing this is the 3Shape D900 lab scanner. In spite of this, some types of restorations still cannot be done digitally because they require a physical model, which must be fabricated $[9,13]$.

Studies examining the accuracy of different digital impression systems have produced varying and controversial results [14-24]. Kim et al. [14] found that conventional impressions are more accurate than digital impressions made by the iTero system. Moreover, Loos et al. [15] found that covering preparations with metal oxide powder prior to scanning alters the geometry of the surface and that may compromise the internal fit of the restoration. On the other hand, Ender et al. [16] stated that digital impressions by direct intraoral scanning circumvents the need to take a conventional impression and pour up a stone model, eliminating two of the steps that may influence the accuracy and precision of the final restoration. Likewise, Syrek et al. [17] found that restorations created from Lava C.O.S. intraoral scans had significantly better marginal fits than restorations made using the conventional impression technique. Additionally, Luthardt et al. [18] established that indirect data acquisition through scanning a conventional impression resulted in better marginal and internal fit, compared with direct intraoral scanning.

There has been much controversy surrounding the accuracy of digital impression systems due to the limitations related to data gathering. Hence, to supplement the current body of literature, this in-vitro study compared the accuracy of digital impressions from a selected subset of these CAD/CAM systems. The null hypothesis of the study stated that there is no significant difference between the accuracies of these particular digital impression systems.

\section{Materials and Methods}

This study was conceived to test the accuracy of digital impression systems. The study design was set according to the ISO/FDIS 12836 Annex B (Bridge shaped specimen for form reproduction testing) [25]. Figure 1 is a flowchart of the study.

The sample size $(n=5)$ for each group of this study was determined based on the results of a pilot study. The sample size calculation was done at a 0.05 level of significance and power of $90 \%$ using nQueri software version 17.0.

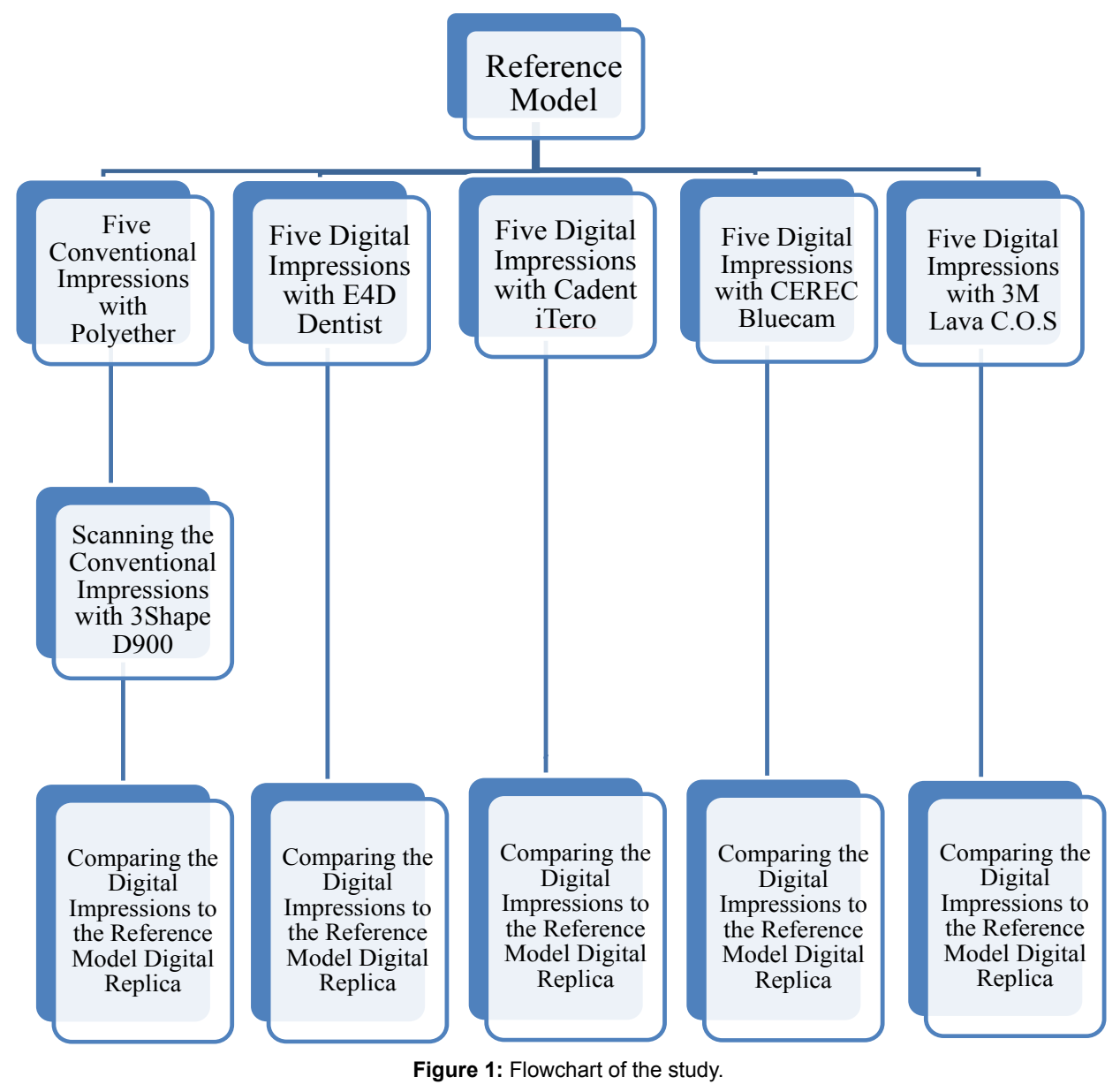




\section{Reference model fabrication}

A typodont model (D95SDP-200, Kilgore International, Inc., Coldwater, MI) with a missing mandibular left first molar was prepared for a three-unit bridge. Axial and occlusal reduction was accomplished by rotating a coarse chamfer diamond bur (KD7W6, Brasseler USA, Savannah, GA) against the abutment teeth. The chamfer bur was selected to create a modified chamfer finish line and provide an optimal preparation for interpretation by the scanning device. Using water spray with the cutting instrument, the operator reduced the axial surface to a depth of 1 to $1.5 \mathrm{~mm}$. Then, the coarse diamond bur was replaced with a fine bur (KD7W6, Brasseler USA, Savannah, GA) to smoothen the surface of the preparation. After the preparation was completed and cleaned, an impression of the prepared teeth was made using polyether impression material (Impregum Penta Soft Quick Step, 3M ESPE, Seefeld, Germany). Prior to taking the impression, polyether tray adhesive (3M ESPE, St. Paul, MN) was applied to the internal surface of the tray. This was done to retain the material in the tray while taking the impression and pouring the reference model. Afterward, the impression was beaded and boxed with wax and poured with extra-hard dental epoxy resin material (EP85-215 dental epoxy, Eager Polymer, Chicago, IL) for fabrication of the reference model. The two liquids were initially hand mixed in a 10:1 ratio. Mixing was then completed in a vacuum mixer for 30 seconds. The epoxy resin was poured into the impression on a vibrator and allowed to set overnight, per the instructions of the manufacturer. The epoxy resin reference model was recovered from the impression, trimmed, smoothed, and evaluated under a stereomicroscope (SZ-PT, Olympus, Tokyo, Japan) to remove any attached particles and to highlight the finish line.

\section{Producing the digital reference model}

A digital version of the reference model was needed to subsequently evaluate the accuracy of the digital impressions. To generate the digital reference model, the epoxy resin reference model was scanned five times in a 3ShapeD900 lab scanner (Software Version 2.8.8.8, 3Shape, Copenhagen, Denmark), which was chosen because it was previously demonstrated as accurate to 6 micrometers $(\mu \mathrm{m})$ [26]. The scans were then compared to each other within 3Shape Convince software (Software Version 2.8.8.8, 3Shape, Copenhagen, Denmark); the areas beyond the finish line were removed to limit the analysis to the abutments only. This was accomplished by designating one of the five model scans as the standard scan and the remaining four scans as the floating test scans. The software calculated the difference in spatial measurements between the standard scan and the individual floating test scans, presenting the results in mean, standard deviation, and root mean square. Afterward, a different model scan was then specified as the standard scan and was assessed against the others as the floating test scans. The process was repeated until all five model scans were examined as the standard scan. Each model scan was compared to the others using the differences in mean and the standard deviation values. Based on these criteria, the scan of the epoxy resin reference model that varied the least from the rest of the scans (least mean difference) was selected as the digital reference model to be used for all further comparisons in this study.

\section{Digital impression scanning}

Each system was used to obtain five digital impressions of the epoxy reference model. The epoxy resin reference model was first scanned by the E4D Dentist (Software Version 4.2, E4D Technologies, Richardson, TX) and the Cadent iTero digital impression systems (Software
Version 4.5, Align Technology, Inc., San Jose, CA) because they did not require surface powdering. In order to test the 3Shape D900 lab scanner (Software Version 2.8.8.8, 3Shape, Copenhagen, Denmark), conventional impressions of the epoxy resin reference model were first required. These tangible impressions were made using polyether impression material (Impregum Penta Soft Quick Step, 3M ESPE, Seefeld, Germany) and were subsequently scanned by the 3Shape D900 system. This is the same system that was used to make the digital reference model and has the capability to create digital impressions also. The epoxy resin reference model was then scanned with the 3M Lava C.O.S. (Software Version 2.0, 3M ESPE, St Paul, MN) and the CEREC Bluecam systems (Software Version 4.2.0, Sirona Dental Systems GmbH, Bensheim, Germany), which sprayed their respective surface powders onto the model. The reference model was cleaned of the surface powder and dried with water and air spray in between the use of those two systems. Each of the scanning procedures was conducted according to the instructions specified by their respective manufacturers. All of the digital and conventional impressions were performed by the same operator. The processed data (output data) set was evaluated without any further manipulation beyond a manufacturer's instructions for the intended end user.

\section{Testing the accuracy of the digital impressions}

After the digital impressions were completed, three-dimensional surface model files were obtained from the manufacturers in .stl format to measure them against the digital reference model. All comparisons were made using 3Shape Convince software (Software Version 2.8.8.8, 3Shape, Copenhagen, Denmark), which works by minimizing distance criteria. The number of points in the point cloud from the digital reference model reading was calculated, and then the digital reference model file was aligned to each three-dimensional surface model file. Utilizing color-difference maps, the spatial discrepancies between the digital reference model and the three-dimensional surface models were identified and analyzed (Figures 2 and 3). The test sensitivity was adjusted to detect differences from 1 to $500 \mu \mathrm{m}$. The comparison between the computer files was limited to the area of tooth preparation between the two abutment teeth, and therefore, all areas beyond the finish line were ignored. The 3Shape Convince software presented the results in numerical form (number of comparison points, minimum, maximum, root mean square, means, standard deviation and tolerance). The same operator handled all of the comparisons in the software (Figures 2 and 3).

\section{Statistical analysis}

The analysis of the data was accomplished using SPSS software version 21.0, which includes descriptive analysis (Means, Standard

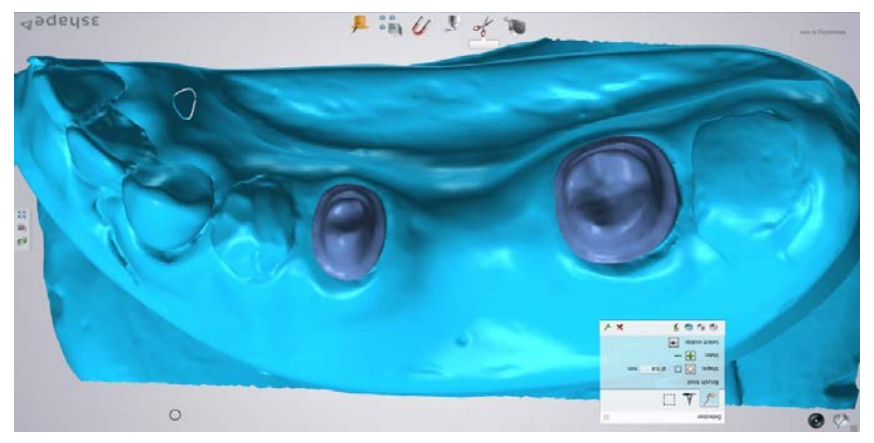

Figure 2: Selection of comparing areas. 


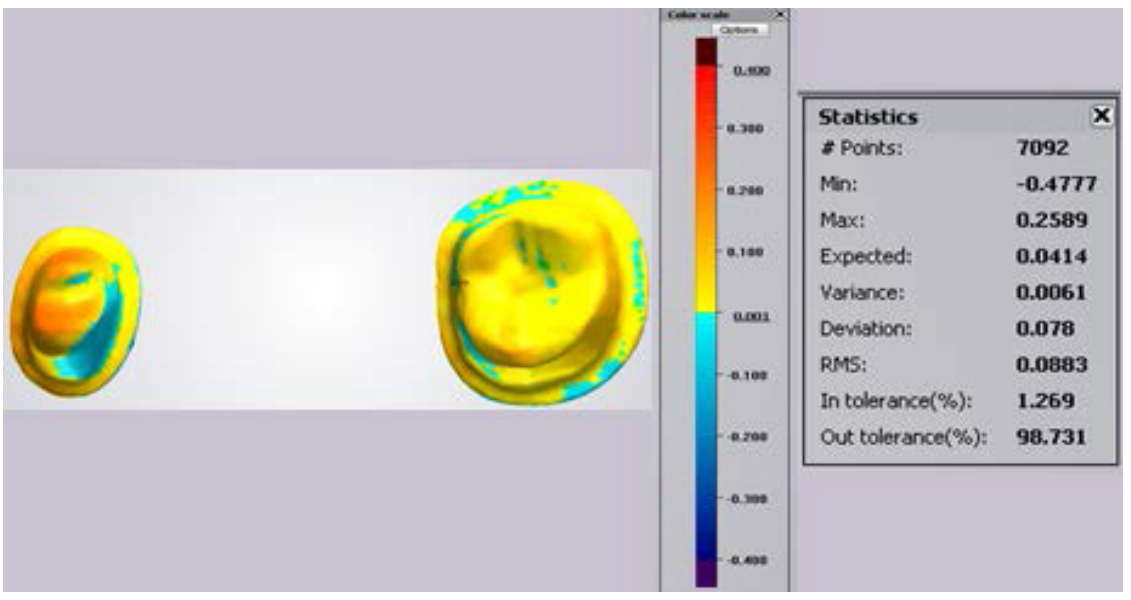

Figure 3: Result of digital impression comparison to the digital reference model.

Deviations, Standard Errors, Confidence Intervals), One Way ANOVA, and Multiple Comparisons Post-Hoc tests via Tukey's HSD.

\section{Results}

The results of the comparison between the digital impressions and the digital reference model were presented to the operator in root mean square, using micrometers.

Thus, the mean difference (standard deviation) values of the different groups were the following: Cadent iTero - 23 (3) $\mu \mathrm{m}, 3 \mathrm{M}$ Lava C.O.S. - 36 (19) $\mu \mathrm{m}$, 3Shape D900 - 44 (18) $\mu \mathrm{m}$, CEREC Bluecam - 68 (12) $\mu \mathrm{m}$, and E4D Dentist - 84 (4) $\mu \mathrm{m}$. Based on these findings, the Cadent iTero system was the most accurate, followed by $3 \mathrm{M}$ Lava C.O.S. The E4D Dentist system was the least accurate among all the tested systems. Table 1 consists of the mean differences and standard deviations of the study groups.

Since the outcomes were continuous variables with more than two testing groups, the data sets were tested for significance using One Way ANOVA. The assumption of equal variances was assessed via Levene's test, and it was considered tenable if the p-value was more than 0.05 . Data sets were tested for normality using Q-Q plots, histograms, and Kolmogorov-Smirnov tests. The p-values for all the groups with Kolmogorov-Smirnov tests were more than 0.05 . Therefore, the data sets were considered normally distributed.

The p-value from Levene's test was greater than 0.05 ; hence, there was no significant evidence that the assumption of equal variances was violated. The test results of One Way ANOVA indicated that the difference between groups was significant $(\mathrm{p} \leq 0.001)$. Multiple comparisons post-hoc tests showed that the E4D Dentist system was significantly different from Cadent iTero, $3 \mathrm{M}$ Lava C.O.S., and 3ShapeD900. Also, CEREC Bluecam exhibited significant differences from Cadent iTero and 3M Lava C.O.S. At the same time, there were no significant differences among $3 \mathrm{M}$ Lava C.O.S., Cadent iTero, and 3 ShapeD900. Table 2 contains the results of the multiple comparisons post-hoc tests. Figure 4 shows bar charts with standard errors of the study groups.

\section{Discussion}

This study was arranged to investigate the accuracy of CAD/CAM systems in terms of digital impression taking. Using the results of an earlier pilot study with the same systems and methodology was a more reliable and scientifically sound method to calculate appropriate sample size for this study, as opposed to relying on the results of previous studies that were completed in different conditions. The sample size was calculated by a professional statistician, who worked with nQuary software version 17.0. The sample size of $n=5$ per group was calculated to be sufficient enough to obtain a power of $90 \%$ and Type I error rate of $\alpha=0.05$.

In this study, several digital impressions were collected from five CAD/CAM systems. These were superimposed on a virtual replica of a reference model (digital reference model) that was acquired from a lab laser scanner accurate to $6 \mu \mathrm{m}$ [25]. The comparisons showed that digital impressions made using Cadent iTero, with a mean difference (and standard deviation) of 23 (2) $\mu \mathrm{m}$, were the most accurate. This was followed by $3 \mathrm{M}$ Lava C.O.S. with 36 (19) $\mu \mathrm{m}$, 3Shape D900 with 44(18) $\mu \mathrm{m}$, and CEREC Bluecam with 68(12) $\mu \mathrm{m}$. E4D Dentist digital impressions were the least accurate, with a measurement of $84(4)$ $\mu \mathrm{m}$. The differences among the systems altogether were statistically significant, according to One Way ANOVA analysis. Thus, the null hypothesis was rejected in favor of the alternative hypothesis.

These results could be due to the fact that, unlike $3 \mathrm{M}$ Lava C.O.S. and CEREC Bluecam, the Cadent iTero system does not require surface spraying with powder. While the E4D Dentist system also does not require surface spraying, it is possible that the reason that its digital impressions were much less accurate than those made by the Cadent iTero system is due to the data capturing techniques that they use. The Cadent iTero system wand uses a laser confocal point and stitch method, while the E4D system uses laser high-speed point and stitch. Comparing the Cadent iTero to the 3Shape D900 digital impressions showed that the difference was not significant, however. Regardless, the Cadent iTero scans were more accurate, a difference that can be possibly attributed to dimensional changes of the conventional impression materials, distortion of the conventional impression scan by the 3Shape D900 system, or a combination of both factors.

Elastomeric impression materials are considered the gold standard for impression accuracy in fixed prosthodontics. According to ADA Specification No. 19, elastomeric impression material should be able to reproduce details as fine as $25 \mu \mathrm{m}$ to be considered accurate [27]. However, the testing methodology set by the ADA is different than the one followed in this study. The ADA method tests the linear change in the impression materials between two fixed points in two dimensions. 


\begin{tabular}{|c|c|c|}
\hline Systems & Mean Differences $(\boldsymbol{\mu m})$ & Standard Deviations $(\boldsymbol{\mu m})$ \\
\hline 3M Lava C.O.S. & $36 \mu \mathrm{m}$ & $19 \mu \mathrm{m}$ \\
\hline CERECBluecam & $68 \mu \mathrm{m}$ & $12 \mu \mathrm{m}$ \\
\hline E4D Dentist & $84 \mu \mathrm{m}$ & $4 \mu \mathrm{m}$ \\
\hline 3Shape D900 & $44 \mu \mathrm{m}$ & $18 \mu \mathrm{m}$ \\
\hline Cadent iTero & $23 \mu \mathrm{m}$ & $3 \mu \mathrm{m}$ \\
\hline
\end{tabular}

Table 1: Mean differences and standard deviations of the study groups.

\begin{tabular}{|c|c|}
\hline Systems & Significant Difference $(\mathbf{p}<\mathbf{0 . 0 5})$ \\
\hline & Cadent iTero \\
\hline E4D Dentist & 3M Lava C.O.S. \\
& 3Shape D900 \\
\hline CERECBluecam & Cadent iTero \\
& 3M Lava C.O.S. \\
\hline
\end{tabular}

Table 2: Results of multiple comparisons Post Hoc tests between digital impression systems.

\section{Bar Charts with Standard Error}

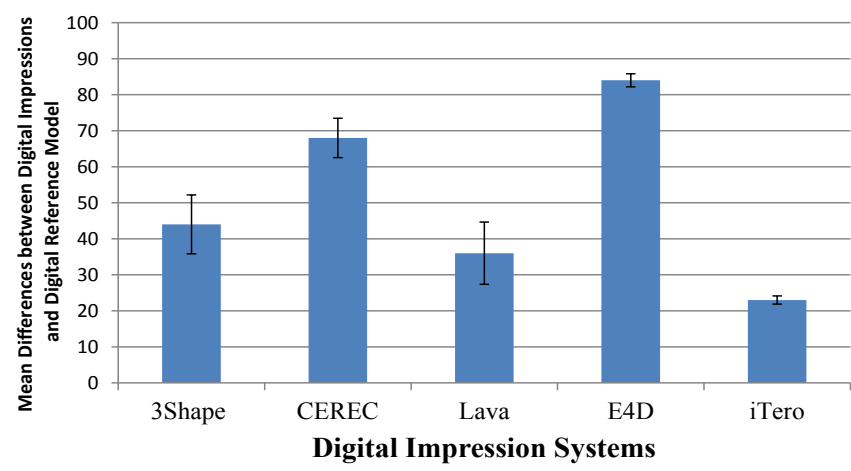

Figure 4: Bar chart with standard error of the study groups.

In this study, the accuracy of the digital impression systems was evaluated in three dimensions. The incongruity between the two testing methods makes it difficult to determine whether this study's results are acceptable by ADA standards.

The results of this study can be compared to the findings of previous research in the literature. The results were in agreement with the work of Ender et al., who evaluated the accuracy of digital impressions from CEREC, iTero, and Lava. They found that the iTero system was the most accurate to $35 \mu \mathrm{m}$, followed by Lavato $45 \mu \mathrm{m}$ and CEREC to 52 $\mu \mathrm{m}$ [18]. Van der Meer et al. [20] compared digital impressions made using iTero, Lava, and CEREC. The investigators actually found that Lava was the most accurate, followed by iTero and CEREC. However, similar to this study, the difference between iTero and Lava was not significant, while the difference between those two systems and CEREC was significant [19]. Sorensen tested the accuracy of full arch scanning with the E4D system and found that the digital impression greatly exaggerated the posterior, anterior, and mesiodistal dimensions of the arch. In that study, a lab scanner was used to make virtual models of conventional impressions for the CAD/CAM production of final restorations. The results of the conventional impression scanning were not significantly different from other testing systems [21]. That finding is in disagreement with the study by Quaas et al. [22], who found that conventional impression scans were less accurate and cannot be recommended as a data acquisition method for CAD/CAM restorations. The contradiction in the results of the two studies could be because Quaas et al. scanned the impressions with a mechanical touch probe scanner, which might have distorted the impression surface. In contrast, this current study used a digital scanner similar to that in the study by Sorensen, which does not apply any pressure on impression surfaces. In addition, Guth et al. [23] found that digital impressions made using Lava C.O.S. were more accurate than a laboratory scan of a conventional impression and conversion to a digital file. Guth et al. used the same impression material as this study, but they implemented a different lab scanner.

Admittedly, this study had its own limitations. The reference model was made of an epoxy resin material that has different surface reflections than human dentine, so the results could differ if natural teeth were used. Moreover, it was possible to scan the reference model using the CEREC Bluecam and 3M Lava C.O.S. systems without the application of a surface powder that adds a fine layer. Nonetheless, the surface spray was still applied before the digital impressions were taken, in accordance with the recommendations of the manufacturers. Also, the impressions for this study were taken in conditions that are less challenging than actual mouths of patients, and factors such as patient movement, saliva, and mouth opening may make an impression less accurate. Flugge et al. [24] tested the accuracy of the iTero digital impression system intra orally and extra orally and found that the extra oral scans were more accurate.

Since the completion of this study, there have been technological advances in the systems tested, including software and hardware upgrades. Therefore, the results of this study could potentially differ from data gathered using the more recent versions of the systems. Nevertheless, CAD/CAM systems also involve processing steps, such as scanning, which are partially dependent on the operator's skill and may therefore create some limitations.

With this study's limitations in mind, further laboratory studies, randomized clinical trials, and prospective clinical studies are needed to test the accuracy of the systems that were examined. Future investigation must address the quality of digital impressions, specifically improving data capturing technique and the use of different surface scanning materials (powders and liquids) to enhance the accuracy of the acquired digital impressions.

\section{Conclusions}

Within the limitations of this study, the following conclusions were drawn:

1) There were statistically significant differences between the accuracy of the digital impressions obtained from these systems. This is most evident when comparing the systems that were the least accurate, such as CEREC Bluecam and E4D Dentist, with those that were on the higher end of the accuracy spectrum.

2) Digital impressions from the Cadenti Tero system were the most accurate.

\section{References}

1. Wang CJ, Millstein PL, Nathanson D (1992) Effects of cement, cement space marginal design, seating aid materials, and seating force on crown cementation J Prosthet Dent 67: 786-790.

2. Wolfart S, Wegner SM, Al-Halabi A, Kern M (2003) Clinical evaluation of marginal fit of a new experimental all-ceramic system before and after cementation. Int J Prosthodont 16: 587-592.

3. Kenyon BJ, Hagge MS, Leknius C, Daniels WC, Weed ST (2005) Dimensiona accuracy of 7 die materials. J Prosthodont 14: 25-31.

4. Chen SY, Liang WM, Chen FN (2004) Factors affecting the accuracy of elastometric impression materials. J Dent 32: 603-609. 
Citation: Ali AO (2015) Accuracy of Digital Impressions Achieved from Five Different Digital Impression Systems. Dentistry 5: 300. doi:10.4172/21611122.1000300

5. Bloem TJ, Czerniawski B, Luke J, Lang BR (1991) Determination of the accuracy of three die systems. J Prosthet Dent 65: 758-762.

6. Luthardt RG, Kühmstedt $\mathrm{P}$, Walter MH (2003) A new method for the computeraided evaluation of three-dimensional changes in gypsum materials. Dent Mater 19: 19-24.

7. Miyazaki T, Hotta Y, Kunii J, Kuriyama S, Tamaki Y (2009) A review of dental CAD/CAM: current status and future perspectives from 20 years of experience. Dent Mater J 28: 44-56.

8. Birnbaum NS, Aaronson HB (2008) Dental impressions using 3D digital scanners: virtual becomes reality. Compend Contin Educ Dent 29: 494, 496 498-505

9. Birnbaum N, Aaronson HB, Stevens C (2009) 3D digital scanners: a high-tech approach to more accurate dental impressions. Inside Dentistry 5: 70-74.

10. Hunter AJ, Hunter AR (1990) Gingival margins for crowns: a review and discussion. Part II: Discrepancies and configurations. J Prosthet Dent 64: 636642

11. Sailer I, Fehér A, Filser F, Gauckler LJ, Lüthy H, et al. (2007) Five-year clinical results of zirconia frameworks for posterior fixed partial dentures. Int $J$ Prosthodont 20: 383-388

12. Reich S, Wichmann M, Nkenke E, Proeschel P (2005) Clinical fit of all-ceramic three-unit fixed partial dentures, generated with three different CAD/CAM systems. Eur J Oral Sci 113: 174-179.

13. Miyazaki T, Hotta $Y$ (2011) CAD/CAM systems available for the fabrication of crown and bridge restorations. Aust Dent J 56 Suppl 1: 97-106.

14. Kim SY, Kim MJ, Han JS, Yeo IS, Lim YJ, et al. (2013) Accuracy of dies captured by an intraoral digital impression system using parallel confocal imaging. Int $J$ Prosthodont 26: 161-163.

15. Loos R, Quaas S, Luthardt RG (2005) Accuracy of conventional impression taking compared tointraoral digitizing-A randomized controlled trial. Joint Meeting of the Continental European (CED) and Scandinavian (NOF) Divisions of the IADR.

16. Ender A, Wiedhahn K, Mörmann WH (2003) Chairside multi-unit restoration of a quadrant using the new Cerec 3D software. Int J Comput Dent 6: 89-94.
17. Syrek A, Reich G, Ranftl D, Klein C, Cerny B, et al. (2010) Clinical evaluation of all-ceramic crowns fabricated from intraoral digital impressions based on the principle of active wavefront sampling. J Dent 38: 553-559.

18. Luthardt RG, Loos R, Quaas S (2005) Accuracy of intraoral data acquisition in comparison to the conventional impression. Int J Comput Dent 8: 283-294.

19. Ender A, Mehl A (2013) Influence of scanning strategies on the accuracy of digital intraoral scanning systems. International Journal of Computerized Dentistry16: 11-21

20. van der Meer WJ, Andriessen FS, Wismeijer D, Ren Y (2012) Application of intra-oral dental scanners in the digital workflow of implantology. PLoS One 7: e43312.

21. Sorensen JA (2014) Accuracy of Full-Arch Scanning With Intra-Oral Scanners. Journal of Dental Research 93 (A) special issue 52.

22. Quaas S, Rudolph H, Luthardt RG (2007) Direct mechanical data acquisition of dental impressions for the manufacturing of CAD/CAM restorations. J Dent 35: 903-908.

23. Güth JF, Keul C, Stimmelmayr M, Beuer F, Edelhoff D (2013) Accuracy of digital models obtained by direct and indirect data capturing. Clin Oral Investig 17: 1201-1208.

24. Flugge TV, Schlager S, Nelson K, Nahles S, Metzger MC (2013) Precision of intraoral digital dental impressions with iTero and extraoral digitization with the iTero and a model scanner. America Journal of Orthodontics Dentofacial Orthopedics144: 471-8.

25. Dentistry -Digitizing Devices for CAD/CAM Systems for Indirect Dental Restorations-Test Methods to Assess the Accuracy and Precision. Final Draft International Standard. ISO/FDIS 128362011.

26. Persson A, Andersson M, Oden A, Sandborgh-Englund G (2006) A threedimensional evaluation of a laser scanner and a touch-probe scanner. J Prosthet Dent 95: 194-200.

27. Eames WB, Wallace SW, Suway NB, Rogers LB (1979) Accuracy and dimensional stability of elastomeric impression materials. J Prosthet Dent 42 159-162. 